\title{
COLD FIELD-EMISSION CATHODE NOISE ANALYSIS
}

\section{Alexandr Knápek, Lubomír Grmela, Josef Šikula, Ondřej Šik}

Brno University of Technology, FEEC, Dept. of Physics, 61600 Brno, Czech Republic( $\triangle$ alexandr.knapek@phd.feec.vutbr.cz)

\section{Abstract}

Noise diagnostics has been performed on the cold field-emission cathode in high-vacuum. The tested cold fieldemission cathode, based on tungsten wire with ultra-sharp tip coated by epoxy was designed to meet the requirements of transmission electron microscopy, which uses a small and stable source of electrons. Current fluctuations are reduced by improving the structure and fabrication technology.

Noise was measured both in time and frequency domains, which gives information about current fluctuations and also about charge transport. Mutual correlation between the noise spectral density, extractor voltage and beam brightness was analyzed.

Keywords: cold field-emission cathode, noise diagnostics, epoxy coating.

(C) 2012 Polish Academy of Sciences. All rights reserved

\section{Experimental Setup}

The experimental setup consists of a DC source, intended both for powering the electron jet (acceleration and extractor voltage) and both for powering up an ion pump, which is used for the second-stage chamber's decompression. The measured signal is in fact the current of an Yttrium aluminum garnet (YAG), which is the scintillator screen. The current is preamplified and further sampled. The whole configuration is illustrated in Fig. 1, where $\mathrm{C}_{1}$, $\mathrm{C}_{2}$ are filtering capacitors.

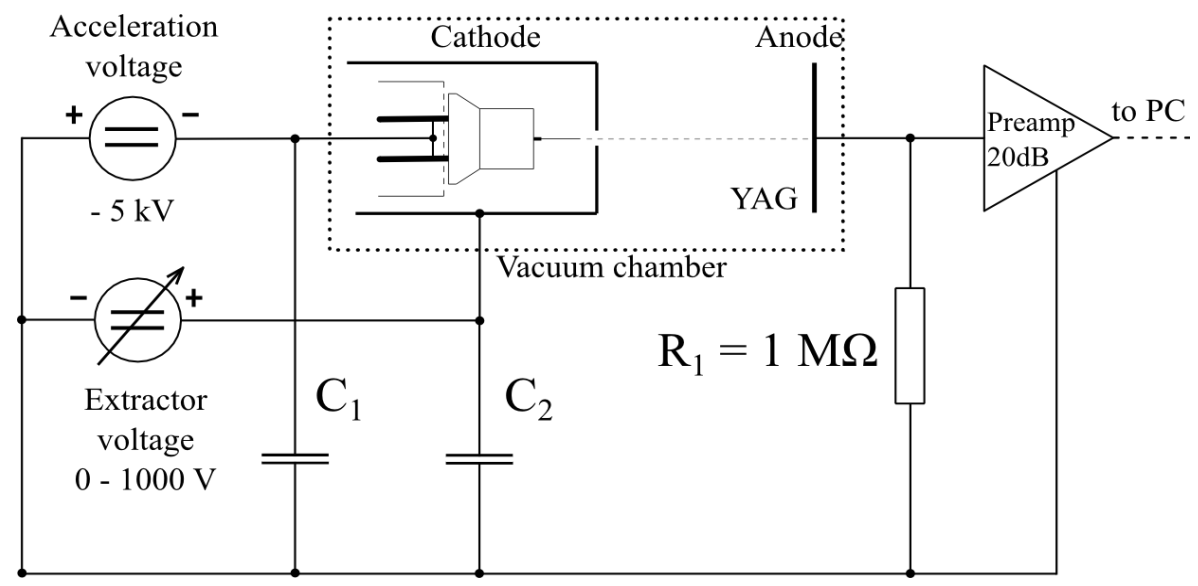

Fig. 1. Schematic illustration of the experimental setup (note that the electron jet is placed in a vacuum chamber).

For the experimental setup, it is necessary to work, at least, under High Vacuum (HV), where the pressure is lower than or equal $1 \times 10^{-6} \mathrm{~Pa}$. Such a low pressure is reached by 
combined means of turbo-molecular and ion-pumps. Ultra low pressure is needed to reduce the amount of ion particles in the chamber, which tend to be attracted back to the cathode surface and bombard its surface, damaging the epoxy layer or the tip itself. It also protects against the high-voltage which could cause a discharge (voltage surge) between the extractor and the cathode; that is very dangerous and could seriously damage equipment and the cathode itself, the gas molecules could be absorbed on the cathode surface, which would lower the cathode current.

\section{Sample Cathode Description}

Pure tungsten wire of the diameter of $0.3 \mathrm{~mm}$ has been used for manufacturing the sample cathode. The fabrication method is based on 2-phase electrochemical etching-method, often called as the Drop-off method, which was firstly described by Melmed [1]. According to the results of the chemical analysis, the wire meets the requirements of copper and mercury-free material, where the particular tungsten (excluding gases) is about $99.95 \%$. The rest of the components are mostly oxygen, molybdenum and carbon, which are present in the amount of about $50 \mathrm{ppm}$ each. Such quantities do not have considerable influence on the cathode's properties, especially on the work function or current stability. The tungsten is present in metallic form, together with some tungsten-residual oxides which are on the cathode's surface. We will describe the influence of the oxide layer on the electric field and tip geometry, which have a crucial role for electron emission, electric field and mainly on current fluctuations.

\subsection{Oxide Layer}

According to our measurements on SEM, the oxide layer possesses an approximately 10 $\mathrm{nm}$ thick homogeneous layer, which covers the cathode surface, including the emission plane on the cathodes' tip. For noise analysis, it is important to mention that the presence of the oxide layer has a direct influence on stabilizing the electron emission, which consequently works, even in a vacuum of $10^{-5} \mathrm{~Pa}$. Fluctuation intensity is not influenced only by the compactness and purity of the oxide layer. Also the oxide thickness has an essential impact on the tunneling-current stability. Taking into account the wavelength of the electron, its mass and velocity, it can be calculated that the width of the depleted layer near the oxide layer is equal to $1 \mathrm{~nm}$. It can be seen that the tungsten-oxide layer acts here as a n-type semiconductor material with $\varphi$ equal to $5.59-5.7 \mathrm{eV}$ [2], therefore it has an essential influence on the stability of the emission current and involves noise mechanisms typical for semiconductor devices. It was reported by Smith [3] that the oxide layer also directly influences the threshold field, while decreasing its intensity. The resin coating affects the emission current by limiting the supply of carriers to the surface, which is strictly dependent on the applied potential, up to the steep rise of the carrier concentration.

\subsection{Relative Light Intensity}

Together with the noise diagnostics, the correlation between the extractor voltage and relative lightning intensity was examined. The lightning intensity has been measured in relative ratio using a CCD camera. For the purpose of lightning evaluation, we have used luminance intensity given by the HSL (hue, saturation, lightness) model. This was obtained from the central maximum created by the electron beam incident on the YAG crystal. 


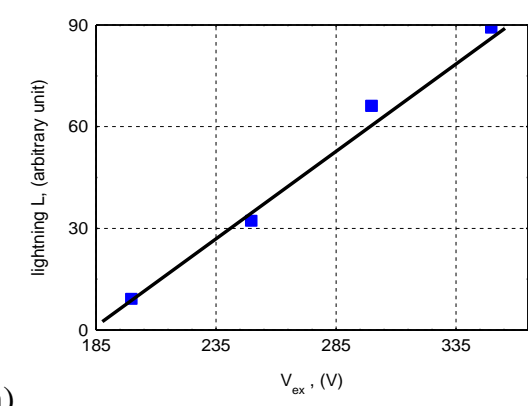

a)

Fig. 2a) Emission intensity (lightning)

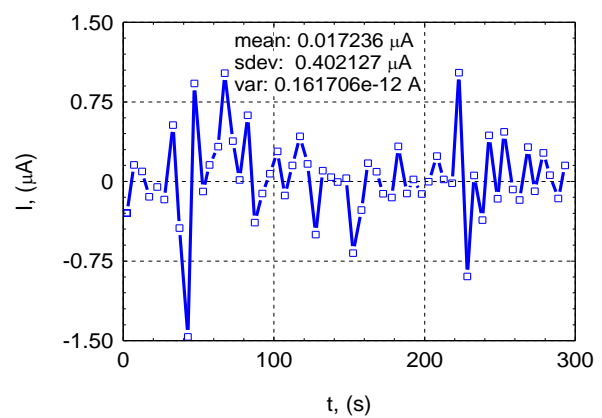

The dependence of lightning on the extraction voltage measured in the incident plane of the YAG scintillator is illustrated in Fig. 2a. As you can see, the lightning is linearly dependent on the extractor voltage. Taking into account that the emitting area is getting smaller as the tip diameter is getting thicker, the electron beam brightness is also rising. The active surface area of the thermoemission cathode has a diameter of approximately $100 \mu \mathrm{m}$, where the electron-emitting tip has less than $0.1 \mu \mathrm{m}$. The value of the electron beam brightness provided by the cold-emission source allows reaching higher current density for the beams of sub-micron diameter. The cold field-emission cathode is also brighter in comparison with the thermoemission cathode and thanks to the low divergence angle (until $1.2^{\circ}$ ) enables to work directly with the virtual source. That can be considered as a great benefit, since it is not necessary to use robust electron optics to focus the beam.

\section{Noise Diagnostics}

The main effort has been focused on the cathode noise diagnostics, in order to describe the transport of the charge carriers flowing from the cathode surface layer. Therefore it was important to identify the main sources of current fluctuation components and to describe their origin. As it was mentioned before, the sample cathode has been tested in a two-stage vacuum chamber under high-vacuum conditions. The accelerating voltage was set to $-5 \mathrm{kV}$, and the extractor voltage was manually increased until the electron emission become optically apparent (we have observed a light spot at a voltage higher than $200 \mathrm{~V}$ ). Immediately after the electron beam appeared on the faceplate of the YAG, the current fluctuation of the cathode was sampled and recorded. Each of the realizations took $480 \mathrm{~s}$ and during this period 80,000 samples were recorded. We have measured the current fluctuation in the time- and frequencydomains. The main effort has been focused on the cathode noise diagnostics, in order to describe the transport of the charge carriers flowing from the cathode surface layer. Therefore it was important to identify the main sources of current fluctuation components and to describe their origin. As it was mentioned before, the sample cathode has been tested in a two-stage vacuum chamber under high-vacuum conditions. The accelerating voltage was set to $-5 \mathrm{kV}$, and the extractor voltage was manually increased until the electron emission become optically apparent (we have observed a light spot at a voltage higher than $200 \mathrm{~V}$ ). Immediately after the electron beam appeared on the faceplate of the YAG, the current fluctuation of the cathode was sampled and recorded. In order to meet the signal quasistationarity requirement for power spectral density analysis, we empirically found the ideal sampling time of a single realization $\mathrm{t}=\mathrm{fs} \times \mathrm{N}=80 \times 103 \times 33.5 \times 106=418 \mathrm{~s}$. To reach good frequency resolution at low frequencies, the sampled signal is processed by the digital quadrature filter bank (DQFB) of order $M=4$, derived from the wavelet transformation algorithm. The output of the DQFB is a set of 16 band-limited fractional signals. From each 
sequence, fractional spectrum Syy, evaluated by the Short-Time Dicrete Fourier Transformation, is received. The resulting Power Spectral Density is calculated by nonparametric Welch's method. The final composition of all fractional spectra is given by equation

$$
S(\omega)=\sum_{m=0}^{M-1} S_{y y}\left(\omega_{m-1}-\omega_{m}\right)
$$

\subsection{Noise Diagnostics in the Time Domain}

The first measurement deals with the current fluctuation analysis in the time domain that had been performed for a time period of $16 \mathrm{~h}$. Emission current fluctuation is shown in Fig. $2 \mathrm{~b}$, where the mean value is $17 \mathrm{nA}$ and the variance $\sigma^{2}=1.6 \times 10^{-13} \mathrm{~A}^{2}$. The variance of the current fluctuation is decreasing with increasing extractor voltage (see Fig. 3a).

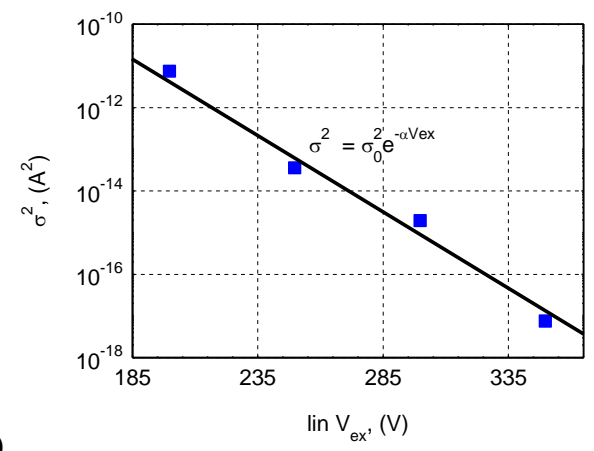

b)

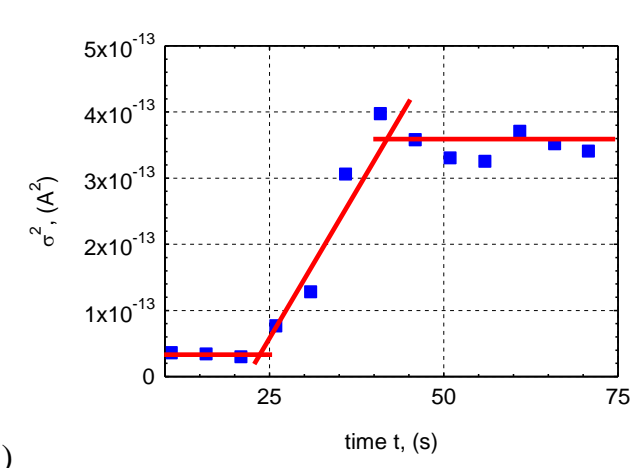

Fig. 3a) Variance of the noise voltage vs. extraction voltage, where $\alpha=2.8 \times 10^{-2} \mathrm{~V}^{-1}, \sigma_{0}{ }^{2}=2.6 \times 10^{-9} \mathrm{~A}^{2}$, $3 b)$ Dependence of the variation on time.

The long-time measurement results (see Fig. 2b) suggest the presence of diffusion, which can be identified from the mean value and standard deviation in time. In general, the extracting potential affects the fluctuating particles, which are following Brown's motion, and can pass through the potential barrier that is lowered due to the applied electric field. Correspondingly, the emission current is slowly increasing. Ions are moving inside the surface layer, causing the creation of a spatial charge which has an essential influence on charge transport and generates burst noise.

With cold field-emission cathodes, the prevailing noise component originates mostly from the local changes on the cathode surface potential. The presence of the adsorbed positive ions of residual gases on the cathode's surface can affect work function. Current fluctuations which are caused by these ions have significant amplitude (higher than 30\%) at very low frequency, especially for cathodes working at room temperature. The noise power spectrum is given by superposition of $1 / f$ and g-r spectra. For g-r, the spectrum variance is constant while for $1 / f$ - noise the variance increases with time, as shown in Fig. $4 \mathrm{~b}$.

\subsection{Noise Diagnostics in the Frequency Domain}

The results of noise diagnostics, performed in the time domain, are further supported by the spectral analysis. According to Fig. 4a, it can be seen that the power spectral density of whole spectra is slowly increasing in time, and that the slope is staying constant. From the slope, which is constantly about 1.5 , it is evident that measured noise has the characteristics of so-called $1 / f$ (flickering) noise.

The $1 / f$-noise is a process with a frequency spectrum such that the power spectral density is proportional to the reciprocal of frequency. 


$$
S_{U}=S_{U(1 H z)} \cdot f^{-1.5} S_{U_{T}}
$$

where $\omega=2 \pi f$ and $\tau=\mathrm{RC}$, from which the noise current density can be computed:

$$
S_{I}=f^{-1.5} S_{U(1 H z)} / R_{L}^{2} \rightarrow \frac{S_{U}}{R_{L}^{2}}\left(1+4 \pi^{2} f \tau^{2}\right),
$$

where $\tau=\mathrm{RC}$. The slope of the function $(n=-1.5)$ implies the presence of diffusion. The whole spectrum is accompanied by the presence of thermal noise, which is expressed in the equation as $\mathrm{V}_{T}$ and is equal to $4 \mathrm{k} T$, where $k$ is the Boltzmann constant and $T$ is the temperature, $300 \mathrm{~K}$. The regression function of the voltage noise spectral density in Fig. $4 \mathrm{a}$ can be mathematically described as

$$
y=a x^{-n}\left(1+b^{2} x^{2}\right),
$$

where $b^{2}$ is the time constant squared and is equal to $\omega^{2} \tau^{2}$. The parameters for the blue curve on Fig. 4a are $a=1.5 \times 10^{-2}$, slope $n=-1.5$ and time constant $b=1 \times 10^{-3} \mathrm{~s}$. According to Fig. 1 we can write

$$
b x=2 \pi \tau=10^{-3} \Rightarrow \tau=R C=160 \mu s .
$$
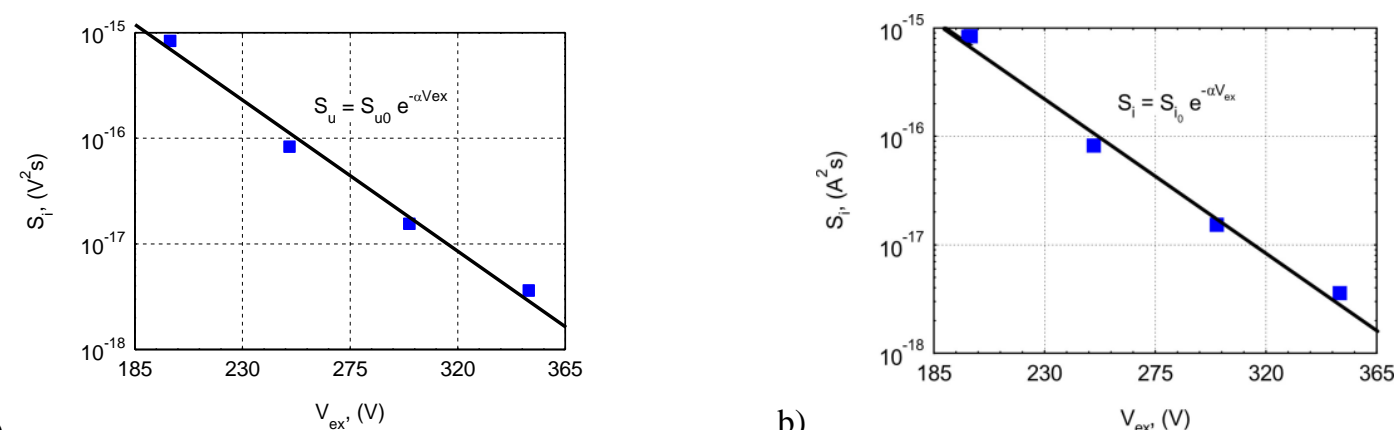

a)

b)

Fig. 4a) Noise spectral voltage density, where $\alpha=2.5 \times 10^{-2} \mathrm{~V}^{-1}, \mathrm{~S}_{\mathrm{io}}=1.2 \times 10^{-13} \mathrm{~A}^{2} \mathrm{~s}$, 4b) Long time noise power spectrum density at $\mathrm{V}_{\mathrm{ex}}=200 \mathrm{~V}$, measured from $t=0 \mathrm{~s}$ (blue) to $t=120 \mathrm{~min}$ (red).

We can see that for the red curve, parameters changed to $a=1.5 \times 10^{-4}$, slope $n=-1.3$ and the time constant remains the same, $b=1 \times 10^{-3} \mathrm{~s}$. In conclusion, the period $\tau$ between each hop to the different energy level is computed, $1.59 \times 10^{-4} \mathrm{~s}$. The cutting frequency is located near $1000 \mathrm{~Hz}$. The $1 / f^{\mathrm{n}}$ noise (where $n>1$ ) originates from the superposition of particular $1 / f$ and generation-recombination (G-R) processes, which originate from adsorption and desorption of various atoms present with some residual gas in the vacuum chamber.

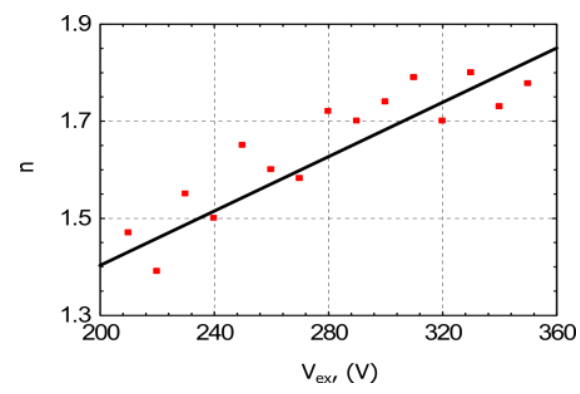

Fig. 5. Dependence of the exponent $n$ of $1 / f$ noise on time.

In the second experiment (see Fig. 5), the function of extractor voltage, which slowly changes, has been observed. According to measured data the noise spectrum is most 
significant around $\mathrm{V}_{\mathrm{ext}}=260 \mathrm{~V}$ and $340 \mathrm{~V}$, thus the lowest fluctuations were observed around $\mathrm{V}_{\mathrm{ext}}=300 \mathrm{~V}$.

\section{Conclusions}

The cathode structure has been deeply examined and described. The results suggest that the quality of the oxide layer has a significant impact on the charge carrier transport and electric threshold field. They are significantly increasing the electric field intensity and affecting electron beam brightness as well. It has been observed that the thin oxide film on the surface acts as a $n$-type semiconductor.

Correlation between the beam light intensity and extractor voltage has been examined as well. It has been discovered that the optimal relation between noise fluctuation level, the light intensity and extractor voltage is close to $\mathrm{V}_{\mathrm{ext}}=300 \mathrm{~V}$.

The ions, which are bombarding the cathode's surface, can be observed from the noise measurement as well. They prove themselves by random burst noise. The bombardment reduces the epoxy layer which leads to its unavoidable damage. The noise spectral density (where the $1 / f$ noise prevails), changes to $1 / f^{\mathrm{n}}$, where $n$ is located between 1 and 2 . In general, the $1 / f^{\mathrm{n}}$ noise originates from the superposition of particular $1 / f$ and generationrecombination (G-R) processes. Therefore the higher the $n$, the more significant G-R is. Generation-recombination is caused mostly by the chemisorption of the ion residuals which are present in the chamber. These effects of chemisorption are unavoidable since only the surface on the tip is protected by the epoxy layer.

\section{Acknowledgements}

This research has been partially supported by the Czech Science Foundation (GACR), under the project No. GAP102/11/0995.

\section{References}

[1] Yu, Z.Q., et al. (2008). Reproducible tip fabrication and cleaning for UHV STM. Ultramicroscopy (online), 873-877.

[2] Raghunandan, S., Jun, H., Jucheol, P., Dong, H.K., Won, B.C. Multistage field enhancement of tungsten oxide nanowires and its field emission in various vacuum conditions. Nanotechnology, 172006, 4840-4844.

[3] Smith, R.C., et al. (2005). Appl. Phys. Lett., 870143111.

[4] Forbes, R.G., Edgcombe, C.J., Valdre, U. Ultramicroscopy, 95, 57.

[5] Slaidinš, I. Accuracy of Noise Measurements for 1/f and GR Noise. Advanced Experimental Methods For Noise Research in Nanoscale Electronic Devices. Springer, 978-1-4020-2169-5, 151, 271-278.

[6] Xu, N.S., Ejaz Huq, S. (2005). Novel cold cathode materials and applications. Materials Science and Engineering, R: Reports, 48(2-5), 47-189.

[7] Diubua, B.C., Chubun, N.N. (1991). Emission properties of Spindt-type cold cathodes with different emission cone material. IEEE Transactions on Electron Devices, 38, 2314-2316.

[8] Sedlák, P., Majzner, J., Šikula, J. (2009). Relation and its validity for piezoelectric ceramics considering temperature. In Proceedings of 20th International Conference on Noise and Fluctuations, Pisa (Italy), 141-144.

[9] Sedlák, P., Majzner, J., Šikula, J. (2011). Noise in Piezoelectric Ceramics at the Low Temperatures. Radioengineering, 20(1), 200-203.

[10] Knápek, et al. (2011). Noise of Cold Emission Cathode. In ICNF 2011: 21st International Conference on Noise and Fluctuations. Toronto, Canada: IEEE, 84-87. 